\title{
Successful Orthotopic Heart Transplantation in Patients with Becker Muscular Dystrophy
}

\author{
(1) Derya Aydın, (1) Eser Doğan, (1) Zülal Ülger, (1) Ertürk Levent \\ Ege University Faculty of Medicine, Department of Pediatric Cardiology, İzmir, Turkey
}

\begin{abstract}
Cardiomyopathy is a major factor contributing to mortality and morbidity in patients with Duchenne and Becker muscular dystrophies (DMD/ BMD), and is therefore among the increasingly important findings. These $X$-linked recessive disorders involve the deficiency or absence of dystrophin in the skeletal muscle as well as the myocardium. This defect brings about changes in the cardiac muscle in three phases: an initial hypertrophic stage, followed by an arrhythmogenic stage, and finally end-stage dilated cardiomyopathy due to increased loss of myocytes. While cardiac involvement can be observed in carriers of BMD and DMD, the incidence of dilated cardiomyopathy is reported to be higher in BMD patients than DMD patients. The only curative treatment option for medically refractory dystrophinopathic end-stage heart failure is heart transplantation. In this report, we present two patients, 14 and 15 years of age, who presented with dilated cardiomyopathy and were diagnosed with muscular dystrophy. One of the patients remains under follow-up with a left ventricular assist device as a bridge-to-transplantation, while the other underwent successful orthotopic heart transplantation.
\end{abstract}

Keywords: Becker muscular distrophy, heart tranplantation, left ventricular asist device

\section{Introduction}

\section{Case 1}

Cardiac muscle involvement is one of the most important causes of death in muscular dystrophies. Heart transplantation and ventricular assist device applications are controversial in these patients due to their neurological prognosis. In this article, 2 cases successfully treated with heart transplantation and VAD applications are presented (1). Permission was obtained from patients to share their medical informations.

\section{Fourteen-year-old boy}

Initial echocardiography performed at age 14 due to complaints of chest pain, coughing, and tiring easily following an upper respiratory tract infection revealed findings consistent with dilated cardiomyopathy (left ventricular ejection fraction [(LVEF) was 24\%]. The patient was referred to our center to undergo testing for possible metabolic and genetic diseases and preparation for transplantation. On physical examination at admission, his body weight was $50 \mathrm{~kg}$ (50 ${ }^{\text {th }}-75^{\text {th }}$ percentile), height was 171 $\mathrm{cm}\left(97^{\text {th }}\right.$ percentile), body temperature was $36.7^{\circ} \mathrm{C}$, heart rate was 98/minimum (min), blood pressure was 93/62 $\mathrm{mmHg}$, respiratory rate was $20 / \mathrm{min}$, and oxygen saturation was $100 \%$. Systemic examination revealed rhythmic $\$ 1$ and S2, grade 2-3/6 systolic murmur, weak peripheral pulses, capillary refill time of 2-3 s, jugular venous distension, normal bilateral respiratory sounds, and palpable liver $2 \mathrm{~cm}$ below the costal margin. Asthenic body type and 
bilateral calf muscle (gastrocnemius) hypertrophy were noted. Upper and lower extremity proximal muscle strength was 4/5 and Gowers' sign was positive. The patient had been born at term by normal spontaneous vaginal delivery (NSVD) with a birth weight of 3,700 gram (g) and had exhibited mental and motor development comparable to his peers. His parents were not consanguineous and his family history was unremarkable. Results of routine tests included white blood cell (WBC) count: 9,350/ $\mathrm{mm}^{3}$, hemoglobin ( $\mathrm{Hb}$ ) level: $13.1 \mathrm{~g} / \mathrm{dL}$, platelet count: 217,000/ $\mathrm{mm}^{3}$, creatine kinase $(\mathrm{CK}):$ 2,161 U/l, troponin: $142 \mathrm{ng} / \mathrm{L}$, and pro-brain natriuretic peptide (pro-BNP): 3,005 pg/mL. Echocardiogram showed dilated cardiomyopathy, increased left ventricular end-diastolic (LVED) diameter, LVEF 20\%, and first-degree mitral regurgitation. Treatment was initiated with digoxin, enalapril, aspirin, spironolactone+thiazide. Due to his elevated muscle enzymes and proximal muscle weakness observed in physical examination, the neurology, metabolism, and genetics departments were consulted regarding muscular dystrophy. Muscle biopsy and DMD gene analysis were ordered. The results of the muscle biopsy were consistent with BMD. Three months after diagnosis, the patient was admitted due to heart failure and an left ventricular assist device (LVAD) was implanted by the cardiovascular surgery team (Image 1).

After 2.5 years of follow-up in the pediatric cardiology outpatient clinic with the LVAD, a suitable donor was found and the patient underwent orthotopic heart transplantation. Post-transplantation ventricle size and functions were normal (LVEF 65\%) and valve functions were normal (Image 2). Antithymocyte globulin, mycophenolate mofetil, and methylprednisolone were given at immunosuppressive doses. Tacrolimus therapy was initiated on postoperative day 3. Ganciclovir and acyclovir

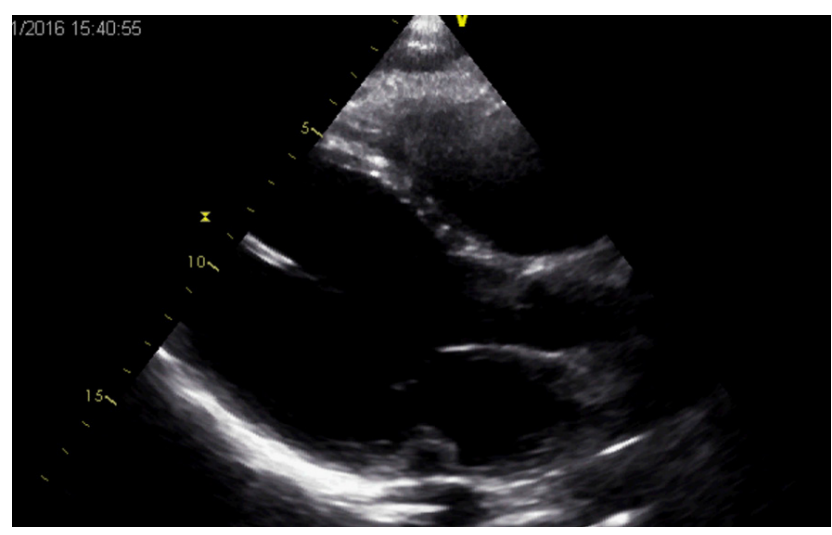

Image 1. Echocardiographic image before heart transplantation. The LVAD cannula placed in left ventricle can be seen

LVAD: Left ventricular assist device prophylaxis against cytomegalovirus and herpes infections, and trimethoprim-sulfamethoxazole prophylaxis against Pneumocystis carinii were added to his treatment. Cardiac biopsy was performed in post-transplantation week 1 to evaluate for acute rejection and findings were consistent with grade 1 acute cellular rejection. Explant biopsy material revealed dilated cardiomyopathy. The patient remains under follow-up in our clinic with no complications.

\section{Case 2}

\section{Fifteen-year-old boy}

The patient had been previously evaluated for muscle disorders due to history of tiring quickly and performing poorly in competitive sports compared to his peers since the age of 3 years, but had not received a definite diagnosis. At the age of 15 , he was diagnosed with dilated cardiomyopathy after presenting with effort dyspnea and stomachache, and was referred to our center. On physical examination at admission, his weight was $57 \mathrm{~kg}\left(25^{\text {th }}-50^{\text {th }}\right.$ percentile $)$, height was $170 \mathrm{~cm}$ ( $75^{\text {th }}$ percentile), body temperature was $36.7^{\circ} \mathrm{C}$, heart rate was 106/min, blood pressure was $103 / 62 \mathrm{mmHg}$, respiration rate was $20 / \mathrm{min}$, and oxygen saturation was $100 \%$. Systemic examination findings included rhythmic S1 and S2, grade 2/6 systolic murmur, normal bilateral respiratory sounds, palpable liver $2 \mathrm{~cm}$ below the costal margin, and bilateral calf muscle hypertrophy. Gowers' sign was positive. Upper and lower limb proximal muscle strength was $3 / 5$. The patient had been born at term by NSVD with a birth weight of 3,200 g and had mental and motor development consistent with his peers. His parents were not consanguineous and his family history was unremarkable except that his older brother had died at age 19 due to DMD. Routine tests revealed WBC: 10.250/ $\mathrm{mm}^{3}, \mathrm{Hb}: 14.2 \mathrm{~g} / \mathrm{dL}$, platelet count: $285,000 / \mathrm{mm}^{3}, \mathrm{CK}: 2.812 \mathrm{U} / \mathrm{I}$, troponin: 234

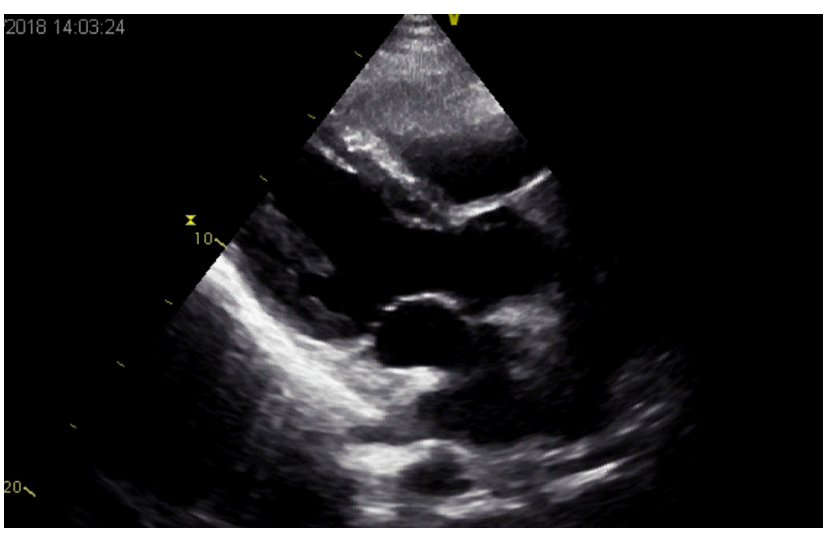

Image 2. Echocardiographic image after heart transplantation. LVED diameters are smaller

LVED: Left ventricular end-diastolic 
ng/L, and pro-BNP: 10,740 pg/mL. Echocardiogram showed dilated cardiomyopathy, increased LVED diameter $(6.5 \mathrm{~cm})$, LVEF $24 \%$, grade $1 \mathrm{MR}$, grade $1 / 2$ tricuspid regurgitation (TR), TR right ventricular systolic pressure: $90 \mathrm{mmHg}$, mean pulmonary artery pressure: $35 \mathrm{mmHg}$, and tricuspid annular plane systolic excursion: $20 \mathrm{~mm}$. Treatment with digoxin, enalapril, aspirin, spironolactone + thiazide therapy was initiated. Due to the patient's elevated muscle enzymes and proximal muscle weakness, the neurology and genetics departments were consulted for muscular dystrophy assessment. Muscle biopsy and DMD gene analysis were ordered. Muscle biopsy revealed sporadic absence of dystrophin consistent with BMD. Results of genetic analysis showed no deletions but duplication between exons 5 and 7 of the dystrophin gene. The patient was diagnosed with BMD based on these clinical, genetic, and pathological findings. Two months after presentation, the cardiovascular surgery team implanted an LVAD due to medically refractory heart failure. The patient has been under follow-up with the LVAD for 2 years in the pediatric cardiology outpatient clinic.

\section{Discussion}

Becker muscular dystrophy affects 1 in 18,450 males; symptoms can appear between the ages of 3 and 21, but the average age at onset is 11 years. Unlike DMD, its progression is slow (2). Mutations responsible for the disease can be deletions or duplications involving any of the 79 exons of the dystrophin gene. Areas of cardiac muscle that lack dystrophin due to these mutations develop myocardial fibrosis, while areas still expressing dystrophin develop compensatory hypertrophy, resulting in local ventricular repolarization heterogeneity and arrhythmias. The development of diffuse fibrosis leads to dilated cardiomyopathy and heart failure.

Although end-stage heart failure is common patients with muscular dystrophy, heart transplantation is not often done. This is due to donor scarcity and the belief that the benefit of transplantation will be limited due to the accompanying myopathy. Moreover, it is widely believed that respiratory muscle involvement will result in longer intubation time and more anesthesia-related complications. Therefore, literature data on transplantation outcomes and pre- and post-transplantation protocols for muscular dystrophy patients are very limited.

There are only a few studies in the literature that report heart transplantation in patients with muscular dystrophy. There are no studies from Turkey on this subject. Cripe et al. (3) reported a successful heart transplantation in a 14-year-old DMD patient 4 years after diagnosis. Rees et al. (4) published 3 cases in which successful orthotopic heart transplantation was performed after a mean follow-up of 40 months. All of these patients tolerated immunosuppression and had no postoperative complications.

BMD patients are considered better candidates for heart transplantation because they have higher rates of dilated cardiomyopathy, longer life expectancy, and better mobility. In a study of 128 children with muscular dystrophy evaluating the frequency of cardiomyopathy and long-term prognosis in $B M D$ and $D M D$, it was determined that the BMD group had more severe MR and larger LVED diameter compared to the DMD group ( $p=0.002)$. The DMD group had a lower 5 -year survival rate than the other groups. Heart transplantation was performed in $25 \%$ of the patients in the study during follow-up (5).

In a large-scale, multi-center study performed at 29 transplant centers between 1990 and 2005, Wu et al. (6) compared 15 BMD patients who underwent heart transplantation with 275 patients who underwent heart transplantation for other reasons and found similar 5-year survival rates. There was also no significant difference between the groups in terms of complications such as infection, rejection, and allograft vasculopathy (6). In another single-center study performed in Spain with 3 BMD patients, intraoperative and postoperative complication rates were no different from those in patients who underwent transplantation for other reasons, but the risk of cyclosporine-induced rhabdomyolysis and secondary myopathy in these patients was emphasized (7).

These studies support heart transplantation as a successful treatment option, particularly in BMD patients who have moderate muscle weakness with no respiratory involvement.

Another option that can be used as an alternative to heart transplantation or a bridge-to-transplantation is the LVAD. Amodeo and Adorisio (8) reported 7 DMD patients who used an LVAD for end-stage heart failure. Three of these patients were lost during the 45-month follow-up period due to infections and hemorrhage. Bleeding, thromboembolic events, and skin infections at the device entry site were recorded as early postoperative complications. The authors of the study reported that LVAD was a life-extending palliative method for patients with refractory end-stage heart failure.

\section{Conclusion}

Understanding the impact of cardiac involvement on mortality and morbidity in patients with muscular dystrophy is important for the development of guidelines for the 
management of cardiomyopathy. Regular neuromuscular and cardiomuscular follow-up and supportive treatment will continue to be therapeutic mainstays until etiologyspecific gene therapies are developed.

As BMD is asymptomatic during the first decade of life, obtaining a detailed family history and conducting biomarker analyses are especially important for early diagnosis.

The only curative treatment option for end-stage heart failure in these patients is heart transplantation. Although few cases have been published to date, long- and short-term outcomes were similar to those in patients who underwent transplantation due to idiopathic dilated cardiomyopathy. Therefore, the available literature data demonstrates that these patients also deserve the opportunity to undergo heart transplantation. In patients for whom a suitable donor cannot be found, the ventricular assist device as a bridge to transplantation is an important option.

\section{Ethics}

Informed Consent: Permission was obtained from patients to share their medical information.

Peer-review: Externally and internally peer-reviewed.

\section{Authorship Contributions}

Design: Z.Ü., Surgical and Medical Practices: E.D., E.L., Literature Search: D.A., Writing: D.A.

Conflict of Interest: No conflict of interest was declared by the authors.
Financial Disclosure: The authors declared that this study received no financial support.

\section{References}

1. Papa AA, D’Ambrosio P, Petillo R, Palladino A, Politano L. Heart transplantation in patients with dystrophinopathic cardiomyopathy: Review of the literature and personal series Intractable Rare Dis Res 2017; 6:95-101.

2. Emery AE. Population frequencies of inherited neuromuscular diseases. A world survey. Neuromuscul Disord 1991; 1:19-29.

3. Cripe L, Kinnett K, Uzark K, Eghtesady P, Wong B, Spicer R. P1.14 Cardiac transplantation in Duchenne muscular dystrophy: a case report. Neuromuscul Disord 2011; 21:645.

4. Rees W, Schüler S, Hummel M, Hetzer R. Heart transplantation in patients with muscular dystrophy associated with end-stage cardiomyopathy. I Heart Lung Transplant 1993; 12:804-7.

5. David M. Sleeper LA, Colan SD, et al. Characteristics and outcomes of cardiomyopathy in children with duchenne or becker muscular dystrophy: a comparative study from the pediatric cardiomyopathy registry Am Heart J 2008; 155:9981005.

6. Wu RS, Gupta S, Brown RN, et al. Clinical outcomes after cardiac transplantation in muscular dystrophy patients. I Heart Lung Transplant 2010; 29:432-8.

7. Ruiz-Cano MJ, Delgado JF, Jiménez $C$, et al. Successful heart transplantation in patients with inherited myopathies associated with endstage cardiomyopathy. Transplant Proc 2003; 35:15131515.

8. Amodeo A, Adorisio R. Left ventricular assist device in Duchenne cardiomyopathy: Can we change the natural history of cardiac disease? Int J Cardiol 2012; 29:161:e43. 